\title{
Calcium Channel Blockers and Esophageal Sclerosis: Should We Expect Exacerbation of Interstitial Lung Disease?
}

\author{
Charalampos Seretis $^{a, b}$ Fotios Seretis $^{a, b}$ \\ George Gemenetzis ${ }^{a, b}$ Nikolaos Liakos $^{a, b}$ \\ Apostolos Pappas $^{c}$ Stavros Gourgiotis ${ }^{a}$ \\ Emmanuel Lagoudianakis $^{a}$ Dimitrios Keramidaris $^{a}$ \\ Nikolaos Salemis ${ }^{a}$ \\ ${ }^{a}$ 2nd Department of Surgery and ${ }^{b}$ Emergency Department, 401 General \\ Army Hospital of Athens, Athens, and 'Internal Medicine Department, \\ Argos Hospital, Argos, Greece
}

\section{Key Words}

Sclerosis - Aspiration - Esophagus - Raynaud syndrome - Nifedipine - Hypoxia Emergency

\begin{abstract}
Esophageal sclerosis is the most common visceral manifestation of systemic sclerosis, resulting in impaired esophageal clearance and retention of ingested food; in addition, co-existence of lung fibrosis with esophageal scleroderma is not uncommon. Both the progression of generalized connective tissue disorders and the damaging effect of chronic aspiration due to esophageal dysmotility appear to be involved in this procedure of interstitial fibrosis. Nifedipine is a widely prescribed calcium antagonist in a significant percentage of rheumatologic patients suffering from Raynaud syndrome, in order to inhibit peripheral vasospasm. Nevertheless, blocking calcium channels has proven to contribute to exacerbation of gastroesophageal reflux, which consequently can lead to chronic aspiration. We describe the case of severe exacerbation of interstitial lung disease in a 76-year-old female with esophageal sclerosis who was treated with oral nifedipine for Raynaud syndrome.
\end{abstract}




\section{Introduction}

Gastrointestinal dysmotility is not uncommon in patients suffering from systemic sclerosis (scleroderma), with a reported incidence even up to $80 \%$ [1]. Although scleroderma may affect all parts of the gastrointestinal tract, esophagus stands for the most frequently invaded organ in cases of gastrointestinal involvement, with gastroesophageal reflux disease (GERD) being the most common consequence of esophageal sclerosis [2]. The main mechanisms through which GERD complicates esophageal sclerosis include impaired efficacy of peristalsis and clearance, reduction of the pressure of the lower esophageal sphincter (LES), high incidence of hiatal hernias due to the gradual shortening of the organ, and delay of gastric emptying [3].

Concerning lung fibrosis and scleroderma, their firm association is well established. Patients suffering from scleroderma are likely to develop interstitial lung disease, accompanied or not by gradual establishment of pulmonary hypertension. Considering the fact that the natural progress of scleroderma is based on the increased accumulation of collagen, which eventually leads to fibrosis, it seems reasonable to assume that in cases of generalized scleroderma invasion, there is a higher risk of developing interstitial lung disease as a consequence of a chronic vicious circle of inflammation and fibrosis [4].

The presence of GERD in scleroderma is a strong contributor to the exacerbation of pulmonary complications, mainly through subclinical microaspiration, which triggers bronchoconstriction and chronic inflammation, highlighting the necessity of aggressive acid-reducing medication support in patients with scleroderma [5]. In addition, these patients should avoid treatment with any drug that could enhance GERD development. Recent studies suggest that calcium channel blockers (CCBs), and particularly nifedipine, increase the risk of GERD by significantly reducing the tone of the LES, increasing esophageal exposure to gastric acid and reducing the amplitude and duration of esophageal peristalsis [6-8]. According to these findings, the administration of CCBs should be avoided, if possible, in patients with GERD.

We report a very interesting case of non-specific interstitial pneumonia developing in a 76-year-old female suffering from esophageal sclerosis and interstitial lung disease, after a 6-month period of receiving oral nifedipine for treating Raynaud syndrome. Our case underlines for the first time the urgent need of considering the potential effect of CCBs as an exaggerator of interstitial lung disease in patients with sclerosis-derived GERD, through enhancing chronic aspiration due to progression of esophageal dysmotility.

\section{Case Report}

Our patient was a 76-year-old never-smoker female who presented to the emergency department complaining of shortness of breath and retrosternal discomfort, after a chocking episode which had awakened her during the night. Physical examination revealed limited thickness of the fingers, presence of ulcers in the oral cavity, palmar telangiectasias and slightly audible crackle sounds bilaterally in the lower respiratory fields. Her vital signs were as follows: blood pressure $160 / 95 \mathrm{~mm} \mathrm{Hg}$, heart rate $110 \mathrm{bpm}$, temperature $37.3^{\circ} \mathrm{C}$, respiration rate $20 / \mathrm{min}$ and $\mathrm{SatO}_{2} 84 \%$. Due to low $\mathrm{SatO}_{2}$ levels, arterial blood gas examination was performed, revealing $\mathrm{PaO}_{2} 51 \mathrm{~mm} \mathrm{Hg}$, $\mathrm{PCO}_{2} 50 \mathrm{~mm} \mathrm{Hg}$ and $\mathrm{pH}$ 7.36. Chest X-ray and electrocardiogram did not reveal any significant 
pathological findings. Blood tests at admission demonstrated leukocytosis $\left(11,800 / \mathrm{mm}^{3}\right)$, slight thrombocytosis $\left(410,000 / \mathrm{mm}^{3}\right)$, C-reactive protein levels of $3.8 \mathrm{mg} / \mathrm{dl}$ and serum lactic dehydrogenase of $412 \mathrm{IU} / \mathrm{l}$. The rheumatological patient's medical history included presence of Sjögren's syndrome, rheumatoid arthritis and cutaneous sclerosis (with clinical regression under treatment) and GERD (under anti-secretory treatment). In addition, she reported that approximately 6 months before she had been diagnosed with Raynaud syndrome and arterial hypertension and since then she had been receiving oral nifedipine $(40 \mathrm{mg})$ daily. The patient mentioned that after the initiation of treatment with nifedipine, arterial hypertension was controlled and she did not experience any other Raynaud phenomenon crisis; nevertheless, she reported signs of gradual intolerance of physical exercise, productive cough episodes and chocking episodes particularly at night, along with exacerbation of GERD symptoms, despite receiving anti-secretory treatment with proton pump inhibitors.

As developing aspiration pneumonia was suspected and since chest X-ray was of no diagnostic value, the patient underwent thoracic CT scanning, which revealed thickening of the esophageal wall, along with bolus retention, and slight intralobular reticular opacity with peripheral distribution in both lungs, with evident signs of chronic interstitial disease (fig. 1). Considering the patient's history, physical examination, laboratory test results and imaging findings, the diagnosis of organizing non-specific interstitial pneumonia was made.

The initial management of our patient in the emergency department consisted of aggressive administration of $50 \% \mathrm{O}_{2}$, salbutamol, ipratropium and budesonide through a Venturi mask and intravenous administration of hydrocortisone and omeprazole. After $2 \mathrm{~h}$, the patient's clinical condition had improved, with a new $\mathrm{SatO}_{2}$ of $93 \%$ and regression of dyspnea. The patient was referred to the Department of Respiratory Medicine for further hospitalization and was discharged after 9 days in excellent clinical condition. It was decided that nifedipine treatment for Raynaud syndrome should be replaced with diltiazem, which has proven to affect less esophageal dysmotility and lower sphincter pressure, compared to other CCBs [9]. Moreover, the daily dose of anti-secretory therapy with omeprazole was doubled. Nearly 12 months after her admission to the emergency department, the patient has not experienced similar severe GERD symptoms or respiratory complications, with both arterial hypertension and Raynaud syndrome efficiently controlled.

\section{Discussion}

CCBs, which are considered to be the gold standard in confronting Raynaud syndrome, have proven to be associated with impaired esophageal motility by various studies, with nifedipine so far dominating the researchers' interest. This adverse effect is mediated by the inhibition of calcium influx into the smooth muscle cells, which is essential for adequate contraction and thus maintenance of efficient pressure of the LES, in order to prevent gastroesophageal reflux [10]. Moreover, CCBs tend to increase esophageal exposure to gastric acid and to reduce the amplitude and duration of esophageal peristalsis [6-8], implying the existence of multiple effects of these drugs on esophageal motility. These findings are in total accordance with the outcomes of a large study by the National Ambulatory Medical Care Service in the United States, which suggested that over the 10-year period of the study, treatment with CCBs was an independent factor for GERD-related physician visits [11]. Consequently, it is evident that CCBs should be prescribed with extreme caution in patients with potentially life-threatening GERD, such as patients with systemic sclerosis, particularly in cases of pre-existing lung invasion of the disease, as chronic aspiration may lead to rapid progression of lung fibrosis. Regarding the possibly beneficial effect of oral CCBs in patients with lung fibrosis, it appears that only short-term positive vascular response can be expected, with these encouraging findings appearing to be limited in interstitial lung diseases that are mainly characterized by inflammation rather than fibrosis, such as in progressive lung sclerosis [12]. 
It is reasonable to assume that treating patients with esophageal dysmotility with CCBs, e.g. for hypertension or angina pectoris, may resemble a serious risk factor of further impairment of GERD and GERD-related diseases, such as chronic inflammation of the respiratory tract. In patients with esophageal sclerosis, this vicious circle of aspiration and lung inflammation, apart from further enhancing fibrosis, is associated with an increased probability of sleep apnea, another life-threatening condition, which is consistent with the feeling of chocking that awakened our patient during the night [13]. Also, the absence of long-term beneficial effect of CCBs in progressive lung fibrosis further highlights the importance of their cautious prescription in patients with esophageal dysmotility. On the other hand, it is suggested that not all CCBs have to the same extent the adverse effects described above; it appears that diltiazem affects less esophageal dysmotility and lower sphincter pressure, compared to other CCBs [9]. This last finding was the reason why in our case was decided not to abandon CCBs, as our patient had experienced severe Raynaud crises. Diltiazem was an attracting alternative, providing minimal damaging effect on GERD and the concomitant aspiration risk. Nevertheless, its administration was accompanied by doubling of the daily proton pump inhibitor dose.

Up to now, there are no solid data that can shed light on this therapeutic challenge. So far, the skepticism regarding the use of CCBs in patients with esophageal sclerosis mainly focuses on investigating the consequences of their administration in esophagus function itself. Although the up-to-date findings are undoubtedly impressive, the final effect of CCBs on GERD-related disorders remains unknown. In our patient, treatment of Raynaud syndrome with nifedipine appeared to have triggered the progression of the underlying interstitial lung disease, which fortunately for our patient was not clinically silent. In the opposite scenario of subclinical progression of the interstitial lung disease, the patient might have developed irreversible respiratory failure or even fatal respiratory arrest due to sleep apnea. Consequently, further prospective studies should be conducted in order to assess the short- and long-term effects of CCBs in patients with scleroderma complicated by esophageal dysmotility, GERD and serious GERD-related conditions, such as interstitial lung disease.

\section{Conclusions}

CCBs have been proven to be associated with a higher risk of developing gastroesophageal reflux. We report a case of rapid progression of interstitial lung disease and exacerbation of gastroesophageal reflux symptoms in a 76-year-old non-smoker female with esophageal sclerosis, after a 6-month period of receiving oral nifedipine. The patient finally addressed the emergency department with symptoms of respiratory distress, which were attributed to organizing interstitial pneumonia. After 9 days of hospitalization the patient was discharged in excellent clinical condition and nifedipine was switched to diltiazem, along with increasing the daily dose of anti-secretory therapy. Nearly 12 months later, the patient has not experienced similarly severe GERD symptoms or respiratory complications and seems to tolerate the new therapeutic approach well. Our case report highlights the necessity of further investigation of the long-term effects of CCBs in GERD-related complications, particularly those imposing a significant risk of mortality, such as in lung fibrosis in 
patients with already impaired esophageal motility, like those suffering from systemic sclerosis.

\section{Disclosure Statement}

The authors declare that they have no competing interests.
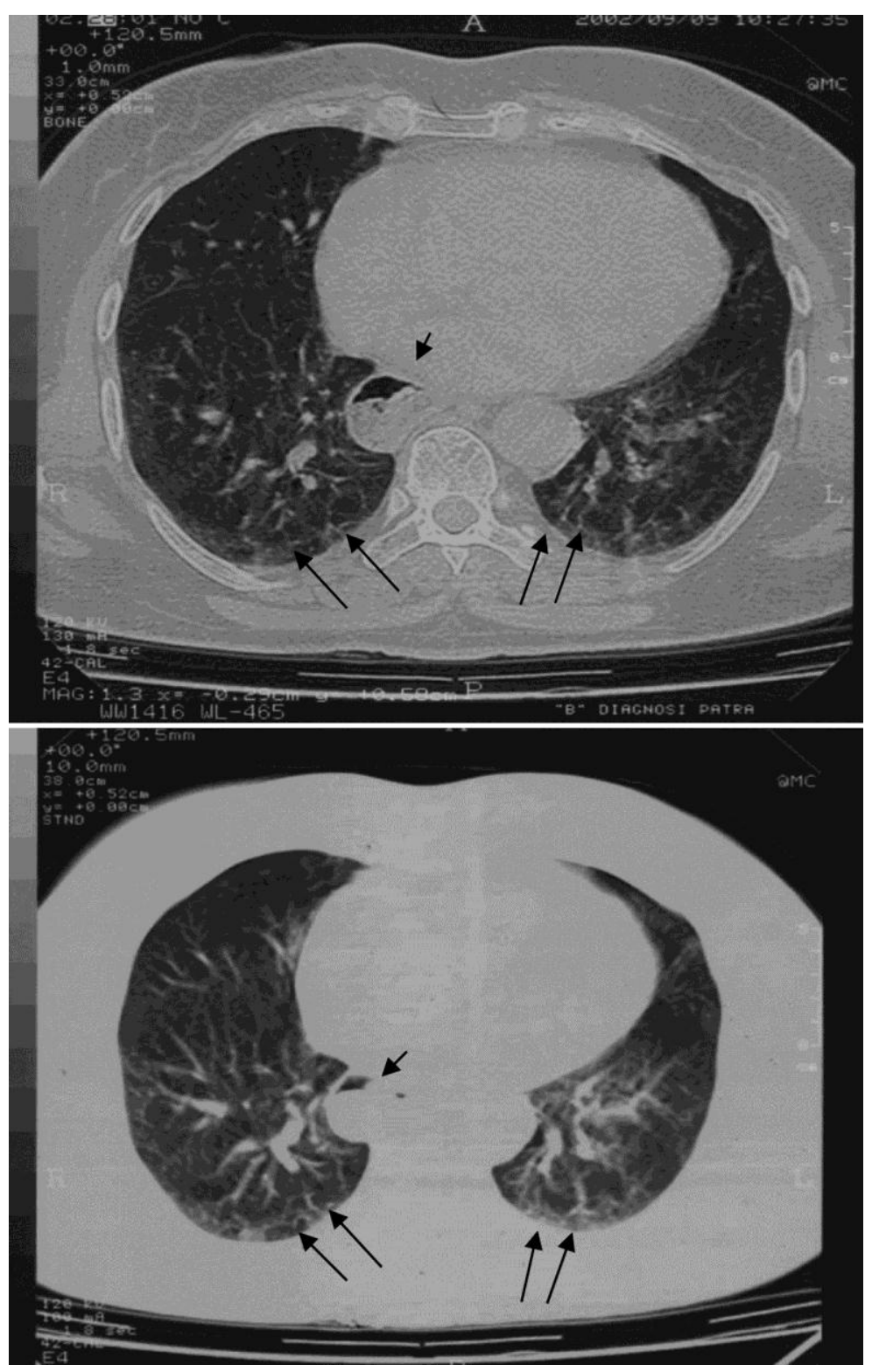

Fig. 1. Thoracic CT imaging: Evident bolus retention in the lumen of the esophagus (short arrows), due to impaired esophageal peristalsis, and slight intralobular reticular opacity with peripheral distribution in both lungs, compatible with interstitial disease complicated by organizing non-specific interstitial pneumonia (long arrows). 


\section{References}

1 Jaovisidha K, Csuka ME, Almagro UA, Soegrel K: Severe gastrointestinal involvement in systemic sclerosis: report of five cases and review of the literature. Semin Arthritis Rheum 2004;34:689-702.

-2 Abu-Shakra M, Guillemin F, Lee P: Exaggerated fibrosis in patients with systemic sclerosis (scleroderma) following radiation therapy. J Rheumatol 1993;20:1601-1603.

3 Ebert EC: Esophageal disease in scleroderma. J Clin Gastroenterol 2006;40:769-775.

4 Mouthon L, Bérezné A, Guillevin L, Valeyre D: Therapeutic options for systemic sclerosis related interstitial lung diseases. Respir Med 2010;104(suppl 1):S59-S69.

5 Bryan C, Knight C, Black CM, et al: Prediction of five-year survival following presentation with scleroderma: development of a simple model using three disease factors at first visit. Arthritis Rheum 1999;42:2660-2665.

-6 Kovac J, Preiksaitis H, Sims S: Functional and molecular analysis of L-type calcium channels in human esophagus and lower esophageal sphincter smooth muscle. Am J Physiol Gastrointest Liver Physiol 2005;289:998-1006.

-7 Ishikawa H, Iwakiri K, Sugiura T, Kobayashi M: Effect of nifedipine administration (10 mg) on esophageal acid exposure time. J Gastroenterol 2000;35:43-46.

8 Yoshida K, Furuta K, Adachi K, et al: Effects of anti-hypertensive drugs on esophageal body contraction. World J Gastroenterol 2010;16:987-991.

9 Hughes J, Lockhart J, Joyce A: Do calcium antagonists contribute to gastro-oesophageal reflux disease and concomitant noncardiac chest pain? Br J Clin Pharmacol 2007;64:83-89.

10 Hamada A, Isii J, Doi K, et al: Increased risk of exacerbating gastrointestinal disease among elderly patients following treatment with calcium channel blockers. J Clin Pharm Ther 2008;33:619-624.

11 Freindenberg F, Hanlon A, Vanar V, et al: Trends in gastroesophageal reflux disease as measured by the National Ambulatory Medical Care Survey. Dig Dis Sci 2010;55:1911-1917.

12 Domenighetti G, Saglini V: Short- and long-term hemodynamic effects of oral nifedipine in patients with pulmonary hypertension secondary to COPD and lung fibrosis. Deleterious effects in patients with restrictive disease. Chest 1992;102:708-714.

13 Prado G, Allen R, Trevisiani V, et al: Sleep disruption in systemic sclerosis (scleroderma) patients: clinical and polysomnographic findings. Sleep Med 2002;3:341-345. 